\title{
CONTRIBUIÇĀO AO ESTUDO DA FLORA DA SERRA DOS ÓRGÃOS ESTADO DO RIO DE JANEIRO, BRASIL I - BIBLIOGRAFIA BOTÃNICA REFERENTE A REGIÃO.
}

\author{
JESUS CARLOS COUTINHO BARCIA \\ Professor do Museu Nacional - UFRJ \\ ODETTE PEREIRA TRAVASSOS \\ Pesquisador do Jardim Botânico - IBDF \\ JOSÉ AQUILES LEAL \\ Ex. Bolsista do CNPq
}

\section{INTRODUÇĀo}

O presente trabalho marca o inicio das publicações do "PROJETO SERRA DOS ORGĀOS" e é a base de outras publicaçōes subseqüentes.

Tal projeto, em desenvolvimento no Departamento de Botânica do Museu Nacional do Rio de Janeiro, tem por finalidade principal o levantamento da Flora, em tôda a sua plenitude, da região denominada "SERRA DOS ÓRGÃOS", situada no Estado do Rio de Janeiro.

Segundo Ruellan (1944), a Serra dos Órgãos abrange os municípios de Nova Iguaçu (Tinguá), Magé (montanhas), Petrópolis, Teresópolis e Nova F riburgo.

Como, para um levantamento de tal amplitude, em uma região já motivo de numerosos estudos botânicos, era necessário consultar vasta bibliografia, tal objetivo tornou-se a Primeira etapa do Projeto.

Hoje, podemos apresentar os primeiros resultados desta fase, com uma bibliografia abrangendo 351 trabalhos onde são citadas plantas mencionadas para a Serra dos Orgãos Ou mesmo aspectos da ecologia e das formaçōes botânicas da Região.

Esperamos que esta primeira contribuição possa ser de alguma utilidade para todos aqueles que se dedicam aos estudos botânicos e mesmo para os que têm especial interêsse na Serra dos Órgãos, um dos maiores complexos biológicos do continente.

\section{SINOPSE}

Obras autores após terem consultado numerosa bilbiografia, apresentam, como resultados, 351

Rio de que fazem referência a plantas ou aspectos botânicos de Serra dos Orgãos, no Estado do Riodriguésia

ANO XXXII - NO 52

Alo de Janeiro 1980 


\section{SUMMARY}

Following intensive bibliographical research, the autors present the resulting bibliography of 351 works that refer to the plants, or botanical features, of the Serra dos Orgäos, in the State of Rio de Janeiro, Brazil.

\section{HISTORICO DAS PESQUISAS BOTÃNICAS NA REGIÃO}

Há mais de 100 anos que grande número de botânicos voltaram suas atençōes para 0 complexo florístico da "Serra dos Orgãos". Por lâ passaram as figuras mais representativas da botânica nos séculos $\mathrm{XIX}$ e XX.

Segundo uma lista elaborada por Rizzini (Flora Organensis), entre os coletores que if trabalharam, podemos citar: A. B. Pereira (1948/1950), Beyrich (1822/1823), Brade (1929/1940), Burchel (1925), Casareto (1839), Gardner (1837/1841), Gaudichaud, Glaziou (1861/1887). Hoehne (1918), Hoehnel (1899, com Ule), Luetzelburg (1910/1916), Land (1833), Riedel (1824/1831), Rizzini (1948/1952), Schenk (1887), Schwacke, Sellow (1814/1831), Ule (1891, 1896/1897, 1899), Wawra (1877), Martius (1817), desses nomes é necessário citar ainda J. Vidal, naturalista do Museu Nacional, grande coletor da Serra dos Órgãos.

Com a criaçäo do Parque Nacional do Serra dos Orgä́os (30.11.1939), cuidou-se de preservar mais a mais a flora e a fauna da regiấo, que ja vinham sofrendo os efeitos danosos da penetraçð̄o indiscriminada pelo elemento humano.

Atualmente, a equipe do "Projeto Serra dos Orgãos", procura fazer um levantamento atualizando os conceitos e fazendo o inventário da flora. Os resultados de tal levantamento serf́o publicados e posteriormente, reunidos em um único trabalho - FLORA DA SERRA DOS ÓRGÃos.

\section{AGRADECIMENTOS}

Desejamos manifestar nossa maior gratidăo aos professores do Departamento de Botånica do Museu Nacional, especialmente ao Dr. Luiz Emygdio de Mello Filho; ao Professor Jorge Pedro Pereira Carauta, do Instituto de Conservação da Natureza; ao Dr. Carlos de Toledo Rizzini, do Jardim Botânico do Rio de Janeiro; Ao Sr. Maurício Braga, bibliotecário do Jardim Botânico do Rio de Janeiro; aos Professores Lucia Ladeira Barcia e Raimundo Paulo de Barros Henriques.

\section{NOTA EXPLICATIVA}

A presente bibliografia é composta de dois índices. No primeiro, em ordem alfabetica de autores, são encontrados os seguintes dados:

1 - Nome do autor

2 - Data

3 - Título do trabalho

4 - Nome do periódico (se houver), número, páginas

5 - Biblioteca onde foram encontrados os trabalhos

No segundo índice, em ordem alfabética de assuntos, encontram-se os números que correspondem ao indice por autores.

\section{CODIGO DAS BIBLIOTECAS}

BMN - Biblioteca do Museu Nacional do Rio de Janeiro

BJB - Biblioteca do Jardim Botânico do Rio de Janeiro

BPSO - Biblioteca do Projeto Serra dos Orgãos. (Localizada na sede do Projeto - Departamento de Botánica do Museu Nacional do Rio de Janeiro.

\section{ABREVIATURAS}

- CNPq. 
An, Soc, Bot. Brasil = Anais da Sociedade Botånica do Brasil.

Annalissouri Bot. Gdn = Annals of Missouri Botanical Garden.

Araucarian. = Annali di Botanica

A $k$. $f$. Botiana-Botânica $=$ Araucariana - Botânica.

Arq. . Bot. = Arkiv for Botanik.

Arq. Bot Est S Paulo = Arquivos de Botànica do Estado de São Pauia

Avq. Jard. Biol. Vegetal = Arquivos do Institu to de Biologia Vegetal.

Ave. Serv. Bot. R. Janeiro = Arquivos do Jardim Botânico do Rio de Janeiro.

Atsoc, Gerv. Flor. = Arquivos do Serviço Florestal.

Atas, Soc, Gr. Brasileiros = Associaçäo dos Gebgrafos Brasileiros.

Ber, dt. Bet, Biol. R. Janeiro = Atas da Sociedade de Biologia do Rio de Janeiro.

B. Inf. Bot. Ges. = Bericht der Deustschen Botanischen Gesellschft-Berlin.

B. Conservaçäo da Natureza. Nat. = Boletim Informativo da Fundaç̋̄o Brasileira para a

8. Mus. Quim. Agricola = Boletim do Instituto de Química Agricola.

B. Mus. Bàol. Nova Sér., Botânica = Boletim do Museu Nacional. Nova Série - Botânica.

B. ES-Brasil.

8. Ser, Nac. Itatiaia = Boletim do Parque Nacional de Itatiaia.

B. Mus, Nac, Pesq. Agron. = Boletim do Serviço Nacional de Pesquisas Agronômicas.

Bradea . Paraense $=$ Boletim do Museu Paraense.

Brittonia = Bradea.

Brasiliana = Brittonia.

Bull. Herb = Brasiliana

Candollea $=$ Boisser. = Bulletin de I'Herbier Boisser.

Contr. Gray = Candollea.

Contr. Gray Herb. Harv. = Contribuitions form Gray Herbarium

Dusenia . S. Nat. Herb. = Contributions from the Unitates States National Herbarium.

Eng. Bot Dusenia.

Fedde Rot JB. = Engler Botanische Jahrbucher.

Hedwiga $=$ Hert. = Feddes Repertorium Specierum Novarum Regni Vegetabilis.

Hook

Kew . Lond. Journ. Bot. $=$ Hooker's London Journal of Botany.

Meded, = Kew Bulletin.

d. Bot. Mus. Herb. Rijks-Univ. Utrecht. $=$ Mededelingen van het Botanisch Museum en

Mem. Herbarium van de Rijks Universiteit Utrecht.

Mem, Inst. Butantan. = Memórias do Instituto Butantan.

Minist, Inst. Oswaldo Cruz $=$ Memorias do Instituto Oswaldo Cruz.

Notizbl. Agrc. Serv. Infor. Agricola = Ministério da Agricultura - Serviço de Informação Agrícola.

Orquide Bot. Gart. Berl. = Notizblatt Botanischen Gartens zu Berlin.

Publs. Field Musquidea.

R Bras,

R Bras, Biol. = Revista Brasileira de Biologia.

Rodriguesiagr, = Revista Brasileira de Geografia.

Symb. Botia = Rodriguesia

Sitzo, Bot, Upsal = Symbolae Botanicae Upsalienses.

A. Kais. Akad. Wiss. Wien., Math.naturw. = Aus den Sitzungs-berichten der Kaiserl $V_{\text {ellozia }}=$ Vellozia

\section{ÍNDICE ALFABÉTICO DE AUTORES E TÍTULOS}

\footnotetext{
1-ABENDROTH, A. - 1961 - Gomesas de Teresópolis. Orquídea, 23 (5):172-175 (BMN) 2

ABENDROTH, A. - 1961 - Apontamentos para as Maxillarias de Teresópolis. Maxillaria picta Hook, e Maxillaria porphyrostele Reichb. f. Orquídea, 23 (5):185-187. (BMN)
} 
3-ABENDROTH, A. - 1966 - Pleuro-thallidinae organensis. Orquídea, 28 (3):193-196 (BMN)

4-ABENDROTH, A. - 1967 - Contribuiçăo para o estudo das Tillandsias em Teresópolis Estado do Rio de Janeiro. Sellowia, 19:109-118 (BMN)

5 - ANDRADE, A. G. de - 1961 - Xiridaceae in CASTELLANOS, A., Os tipos das plant vasculares do Herbário do Museu Nacional. B. Mus. Nac. Nova Sér., Botấnica 283 . (BMN)

6-ARA WJO, P. A. M. - 1950 - Contribuiçăo ao conhecimento da famrlia Asclepiadaceae $\infty$ Brasil. Rodriguesia, 13 (25) $5-221,15$ tabs. (BJB)

7 - ASCHERSON, P. und P. GRAEBNER - 1907 - Potamogetonaceae in ENGLER, A. und K. PRANTL, Das Pflanzenreich, 31:1-184. (BMN)

8-ATALA, F. - 1964 - Ervas daninhas Vellozia 1(4):168. (BPSO)

9 - BARCIA, J. C. C. e S. SOARES - 1977 - Contribuiç5̃o ao estudo das Pteridofitas do Bras Estudos Palinológicos do gênero Polypodium. - I. B. Mus. Biol. Prof. M. Leitão, ${ }^{86}$ 1-5, 10 figs. (BPSO)

10 - BAEHNI, C. - 1938 - Les Ceftis Sud-Americains. Candollea, 7:189-214. (BJB)

11 - BAKER, J. G. - 1870 - Cyatheaceae in MARTIUS, C. F. P. von, Flora Brasiliensis, ${ }^{1}$ (2) :305-334, 591-593, tab. 20 , fig. 1-6, 9-14, 17-19, tab. 21, 22, 40 fig. 1-4, tab. $5^{3}$ $54,67-69$. (BMN, BJB)

12 - BAKER, J. G. - 1870 - Polypodiaceae in MARTIUS, C. F. P. von, Flora Brasiliensis. (2) : $335-610$, tab. 20 fig. $7-8,15,16$ e 20 , tab. $21-39,40$ fig. 5 e 6 , tab. $41-51$ fig. 1 t $^{\prime}$ 6. tab. 55-66 70 (BMN, BJB)

13 - BAKER, J. G. - 1871 - Connaraceae et Ampelidae in MARTIUS, C. F. P. von, Flot Brasiliensis, 14 (2):173-216, tab. 46-52. (BMN, BJB)

14 - BAKER, J. G. - 1873 - Compositae I. Vernoniaceae in MARTIUS, C. F. P. von, Flot Brasiliensis, 6 (2):1-180, tab. 1-50. (BMN, BJB)

15 - BAKER, J. G. - 1876 - Compositae II. Trib. Eupatoriaceae in MARTIUS, C. F. P. von, Flat Brasiliensis, 6 (2):181-376, tab. 51-102. (BMN, BJB)

16 - BAKER, J. G. - 1882 - Compositae III. Asteroideae et Inuloideae in MARTIUS, C. F. $P$ von, Flora Brasiliensis, 6 (3):1-134, t. 1-44. (BMN, BJB)

17 - BAKER, J. G. - 1884 - Compositae IV. Helianthoideae, Helenoideae, Anthemideae, Serf cionideae, Cyranoideae, Ligulatae, Mutissiaceae in MARTIUS, C. F. P. von, Flora Blf siliensis, 6 (3):137-412, tab. 45-108. (BMN, BJB)

18 - BARBOSA, E. S. - 1971 - Catálogo do Herbário do Jardim Botânico do Rio de Janeire Parte I: Alismataceae. Amaryllidaceae, Araceae. Rodrigulsia, 26 (53):297-338. (B.

19 - BARROS, H. e outros - 1958 - Levantamento do Reconhecimento dos Solos do Estado Rio de Janeiro e Distrito Federal. (Contribuiçăo a Carta dos Solos do Brasil). B. Se Nac. Pesq. Agron., 11:1-349. (BPSO)

20 - BARROS, Wanderbilt D. de - 1943 - Relatório do agrônomo W. Duarte de Barros sóbre u excursio à Serra dos Orgãos. Rodriguesia, 7(16) :85-86. (BJB) 
21 - BARROS, Wanderbit D. de - 1952 - Parques Nacionais do Brasil. Minist. Agric. Serv. Inf. Agricola, 1:66-71, 1 tab. (BPSO)

22 - BARROS, Wanderbilt D. de - 1954 - Estudos Botânicos nos Parques Nacionais Brasileiros. Arq. Ser. Flor. 8:233-237. (BJB)

23-BARROSO, G. M. - 1952 - Scrophulariaceae indígenas e exóticas no Brasil. Rodriguesia 15 (27) $: 9-64,44$ tab. (BJB)

24 - BENJAMIM, D. S. - 1962 - Estudo das Aubiacese Brasileiras II. Arq. Jard. Bot. R. Janeiro 18:223-227. (BJB)

25 - BENTHAM. G. - 1859 - Leguimosae L. Papilionacearum: tr. Genisteae, Psolariaceae, Trifolieae, Indigoferae, Galeaceae, Hedysareaceae, Vicieae, Phaseoleae in MARTIUS, C. F. P. von, Flora Brasiliensis, 15(1):1-226, tab. 1-56 (BMN, BJB)

26 - BENTHAM, G. - 1862 - Leguminosae I. Papilionacearum: tr. Dalbergiae et Sophoreae, in MARTIUS, C. F. P. von, Flora Brasiliensis, 15 (1):217-332, tab. 57-127 (BMN, BJB)

27 - BENTHAM, G. - 1870 - Leguminosae II. Swartzieae et Caesalpinieae in MARTIUS, C. F. P. von, Flora Brasiliensis, 15 (2):1-254, tab. 1-66 (BMN, BJB)

28 - BENTHAM, G. - 1876 - Leguminosae III. Mimoseae in MARTIUS, C. F. P. von, Flora Brasiliensis, 15 (2):257-504, tab. 76-138. (BMN, BJB)

29 - BERG, O. - 1857 - Myrtaceae I: Tr. Myrteae in MARTIUS, C. F. P. von, Flora Brasiliensis, 14 (1):1-468, tab. 1-35. (BMN, BJB)

30 - BERG, O. - 1858 - Myrtaceae II. Tr. Barrintonieae, Lecythideae, Granateae in MARTIUS, C. F. P. von, Flora Brasiliensis, 18 (2):469-528, tab. 36-82 (BMN, BJB)

11-BERG, O. - 1859 - Myratacese: Supplementum, utilium elechus in MARTIUS, C. F. P. von, Flora Brasiliensis, 14 (1):529-636 (BMN, BJB)

32 - BiCudo, C. E. M. e R. M. T. BICUDO - 1969 - Algas da lagoa das Prateleiras, Parque Nacional do Itatiaia, Brasil, Rickia, 4:1-40, 75 fig. (BPSO)

33 - BITTER, G. - 1920 - Solana nova vel minus cogniata XVIII. Fedde Repert, 16:91 (BJB)

34-BRADE, A. C. et E. ROSENTOCK - 1931 - Filices Novae Brasilianae II. B. Mus. Nac. $7(3): 135-147,9$ tab. (BMN)

35 - BRADE, A. C. - 1935 - Filices novae Brasilianae IV. Arq. Inst. Biol. Vegetal, 2 (1):1-5, 4 tab. (BJB)

36 - BRADE, A. C. - 1942 - A Composiçăo da Flora Pteridófita do Itatiaia. Rodriguesia, 6 (15):29-43. (BJ8)

37 - BRADE, A. C. - 1942 - Excursão à Serra de Caparaó. Rodriguésia, 6 (15):91-92. (BJB)

38 - BRADE A. C. - 1943 - Labiadas novas do Brasil. Ridriguésia, 7 (16) 27-33, 7 tabs. (BJB)

3 - BRADE, A. C. - 1944 - Begoniaceae do Herbário do Museu Nacional do Rio de Janeiro B. Mus. Nac. Nova Sér. Botânica, 1:1-16, 7 tabs. (BMN)

40 - BRADE, A. C. - 1945 - Contribuiç̃o para o conhecimento da flora dos Parques Nacionais e Serra dos Órgãos. I - Labiatae. Rodriguésia, 9 (19):9-20, 10 tab. (BJB) 
41 - BRADE, A. C. - 1945 - Begònias novas do Brasil - IV. Rodriguesia, 19 (18):23-34, 6 tab. (BJB)

42 - BRADE. A. C. - 1946 - Labiatae e Pteridophyta do Herbário do Museu Nacional do Rio de Janeiro. B. Mus. Nac. Nova Sér., Botânica, 5:1-12. (BMN)

43 - BRADE, A. C. - 1947 - Contribuiçăo para o Conhecimento da Flora do Estado do Espírito Santo. (I - Pteridophyta). Rodriguesia, 10 (21):25-56, 4 est. (BJB)

44 - BRADE, A. C. - 1949 - Relatório de uma excursăo ao município de Passa Quatro, Estado de Minas Gerais. Rodriguesia, 11 e 12 (22 e 23): 133-142. (BJB)

45 - BRADE, A. C. - 1951 - O Gênero "Habenaria" (Orchidaceae) no Itatiaia. (Contribuiçăo para o conhecimento da Flora dos Parques Nacionais do Itatiaia e Serra dos Orgăos. I1). Rodriguesia, 14 (26):7-21, 9 tab. (BJB)

46 - BRADE, A. C. - 1951 - Relatório de uma Excursâo à Serra da Bocaina realizada pelo Naturalista A. C. Brade, de 18 de abril à 24 de maio de 1951. Rodriguesia, 14 (26):55-66, 14 fotos. (BJB)

47 - BRADE, A. C. - 1956 - A Flora do Parque Nacional do Itatiaia. B. Parq. Nac. Itatiaia. $5: 1-85,27$ tab. (BPSO)

48 - BRADE, A. C. - 1965 - Algumas espécies novas do gênero Elaphoglossum (Polypodiacese) da Flora do Brasil. (Filices Novae Brasiliense VII). Arq. Jard. Bot. R. Janeiro, 18:17.23. (B.JB)

49 - BRADE, A. C. - 1965 - Contribuiçăo para o Conhecimento das espécies Brasileiras do Gênero Doryopteris (Polypodiaceae). Arq. Jard. Bot. R. Janeiro, 18:39-72, 18 est. (BJB)P

50 - BRADE, A. C. - 1969 - Algumas espécies novas do gênero Polybotrya da Flora do Brasil. Bradea, 1(2):23-28, 1 tab. (BPSO)

51 - BRADE, A. C. - 1971 - Uma espécie nova do gênero Begonia, do Estado da Bahia, Brasile Sinopse das espécies brasileiras publicadas nos anos de 1944 a 1958 . Bradea, 1(6): 42-43. (PSO)

52 - BRADE, A. C. - 1971 - O Genero Polybotrya do Brasil - I. Bradea, 1 (9):57-67, 6 est(BPSO)

53 - BRADE, A. C. - 1971 - Cyathea sampaiona Brade et Ros. somente uma "Forma" de Cyathea sternbergii Pohl. Bradea, 1(10):73-75, 1 tab. (BPSO)

54 - BRAND, A. - 1901 - Symplocaceae in ENGLER, A. und. K. PRANTL, Das pflanzenreich. 6:1-100 (BMN)

55 - BROTHERUS, V.F. - 1894 - Musci Schenckiani. Hedwigia, 33:127-136 (BMN)

56 - BUREAU, E. et C. SCHUMANN. - 1896 - Bignoniaceae I. in MAR TIUS, C. F. P. von, Flors Brasiliensis, 8 (2):1-230, tab. 69-96. (BMN, BJB)

57 - BUREAU, E. et C. SCHUMANN - 1897 - Bignoniaceae II. in MARTIUS, C. F. P. von, Flor Brasiliensis, 8 (2):229-434, tab. 97-121 (BMN, BJB)

58 - BURRET, M., Die Palmengattung Syagrus Mart., Notizbl. Bot. Gart. Berlin, 13(120):677-696. (BJB) 
- CABRERA, A. L. - 1950 - Notes on the Brasilian Senecioneae. Britonia, 7(2):61. (B.JB).

60 - CARAUTA, J. P. P. - 1968 - Moraceae da Flórula Carioca. Lista das Espécies. Vellozia, 6:32-40 (BPSO)

61 - CARAUTA, J. P. P. - 1969 - Ulmaceae da Fiórula Carioca. Lista das Espécies., A tas Soc. Biol. R. Janeiro, 12 (4):217. (BPSO)

62 - CARAUTA, J. P. P. - 1969 - Urticaceae da Flórula Carioca. Lista das Espécies. Vellozia, 7:54-56 (BPSO)

63 - CARVALHO, J. C. M. - 1968 - Lista das espécies de animais e plantas ameaçadas de extinçäo no Brasil. Fundaç̋̈o Brasileira para Conservaçăo da Natureza, 4: (BPSO)

64 - CASTELLANOS, A. - 1963 - Contribuição para o conhecimento da FIórula da Guanabara. Cactaceae. III. Vellozia, 1(3):104-105. (BPSO)

65 - CASTELLANOS, A. - 1965 - Contribuiç̋o para o conhecimento da Flórula do Itatiaia. Catálogo dos Pteridófitos - Pteridophyta - B. Parq. Nac. Itatiaia, 8 (1):145. (BPSO)

66- CENTRO DE CONSERVAÇĀo DA NATUREZA, Rio de Janeiro. XVI Congresso da Sociedade de Botânica do Brasil. Vellozia, 1 (5):249. (BPSO)

67 - COGNIAUX, A. - 1878 - Cucurbitacese in MARTIUS, C. F. P. von Flora Brasiliensis, 6 (4):1-126, tab. 1-38 (BMN, BJB)

Q - COGNIA UX, A. - 1883 - Melastomataceae I. Microliaceae in MARTIUS, C. F. P. von, Flora Brasiliensis, 14 (3):1-204, tab. 1-48. (BMN, BJB)

69 - COGNIAUX, A. - 1885 - Melastomataceae II. Tibouchineae n MARTIUS, C. P. F. von, Flora Brasiliensis, 14 (3):205-484, tab. 49-108. (BMN, BJB)

70 - COGNIAUX, A. - 1886 - Melastomataceae Ila. Roexieae, Merianiaea, Bertolonieae, Miconieae (pp.) in MARTIUS, C. F. P. von, Flora Brasiliensis, 14 (4):1-212, tab. 1-45. (BMN, BJB)

71 - COGNIAUX, A. - 1887 - Melastomataceae IIb. Miconieae (cont.) in MARTIUS, C. F. P. von, Flora Brasiliensis, 14 (4):205-398, tab. 46-79. (BMN, BJB)

72 - COGNIAUX, A. - 1888 - Melastomataceae IIc. Miconieae (pps), Blakeae, Memecyleae in MARTIUS, C. F. P. von, Flora Brasiliensis, 14(4):397-626. (BMN, BJB)

73-COGNIAUX, A. - 1893 - Orchidaceae I. Cypridelinae, Ophyrydinae, Neottinae (pp.) in MARTIUS, C. F. P. von, Flora Brasiliensis, 3 (4):1-160, tab. 1-34. (BMN, BJB)

74- COGNIAUX, A. - 1895 - Orchidaceae II. Neottinae (pp), Liparidinae, Polystachyinae (pp)in MARTIUS, C. F. P. von, Flora Brasiliensis, 3 (4):157-332, tab. 35-75. (BMN,BJB)

75 - COGNIAUX, A. - 1896 - Orchidaceae III. Polystachynae (pp). Pleurothallidinae (pp) in MARTIUS, C. F. P, von, Flora Brasiliensis, 3 (4):317-494, tab. 75-99. (BMN, BJB)

76 - COGNIAUX, A. - 1896 - Orchidacese IV. Pleurothallidinae (pp) in MARTIUS, C. F. P. von Flora Brasiliensis, 3 (4):493-652, tab. 100-133. (BMN, BJB)

77-COGNIAUX, A. - 1898 - Orchidaceae V. Laelinae (pp) in MARTIUS, C. F. P. von, Flora Brasiliensis, 3 (5):1-188, tab. 1-49. (BMN, BJB) 
78 - COGNIAUX, A. - 1901 - Orchidaceae VI. Laeliinae (pp), Sobraliinae, Phajinae, Cyrto podium (pp) in MARTIUS, C. F. P. von Flora Brasiliensis, 3(5):181-384, tab. 50-81. (BMN, B.JB)

79 - COGNIAUX, A. - Orchidaceae VII. Cyrtopodium pp., Catasetinae, Lycastinae, Gongorinae, Zygopetalinae, Bulbophyllinae, Cymbidiinae in MARTIUS, C. F. P. von, Flora Brasiliensis, 3 (5): 381-624, tab. 82-119. (BMN, BJB)

80 - COGINIAUX, A. - 1904 - Orchidaceae VIII. Maxillariinae, Oncidinae (pp) in MARTIUS, C. F. P. von, Flora Brasiliensis, 3(6):1-202, tab. 1-42. (BMN, BJB)

81 - COGNIAUX, A. - 1905 - Orchidaceae IX. Oncidilnae (pp) in MARTIUS, C. F. P. von, FIora Brasiliensis, 3 (6):197-390, tab. 43-79. (BMN, BJB)

82 - COGNIAUX, A. - 1906 - Orchidaceae X. Oncidiinae (pp), Huntleyinae, Dichaeinae, Sarcan thihae. Adenda et Emendanda. in MARTIUS, C. F. P. von, Flora Brasiliensis, 3 (6):381-588, tab. 80-120. (BMN, BJ8)

83 - COGNIAUX, A. - 1916 - Cucurbitacese: Fevilleae et Melothrieae, in ENGLER, A. und K. PRANTL, Das Pflanzenreich, 66:1-227. (BMN, BJB)

84 - DAU, L., F. SEGADAS-VIANNA e W. T. ORMOND - 1971 - Begoniaceae. Flora Ecológica de Restigas do Sudeste do Brasil, 20:1-23, e tab. (BMN)

85 - DE CANDOLLE, A. - 1861 - Begoniaceae in MARTIUS, C. F. P. von, Flora Brasiliensis, 4 (1):338-388, tab. 91-101. (BMN, BJB)

86 - DE CANDOLE, C. - 1901 - Piperaceae Uleanae e Brasilia. Engl. Bot., JB 29, Beibl. 65:24-27. (BMN)

87 - DE CANDOLE, C. - 1917 - Piperaceae neotropicae III. Notizbl. Bot. Gart. Berl., 7 (62):434-476 (BJB)

88 - DIELS, L. - 1910 - Menispermaceae in ENGLER, A. und K. PRANTL, Das Pflanzenreich, 46:1-345 (BMN)

89 - DOELL, J. C. - 1871 - Gramineae I. Oryzeae et Phalarideae in MARTIUS, C. F. P. von, Flora Brasiliensis, 2 (2):1-32, tab. 1-11. (BMN, BJB)

90 - DOELL, J. C. - 1877 - Gramineae II. Paniceae in MARTIUS, C. F. P. von, Flora Brasiliensis, 2(2):33-342, tab. $12-49$ (BMN, BJB)

91 - DOELL, J. G. - 1878 - Gramineae III. Stipaceae, Agrostideae, Arundinaceae, Pappophoreae, Chlorideae, Avenaceae, Festucaceae in MARTIUS, C. F. P. von, Flora Brasiliensis, 2(3):1-160, tab. 1-43 (BMN, BJB)

92 - DOELL, J. G. - 1880 - Gramineae IV. Bambusa, Hordeacese in MARTIUS, C. F. P. von Flora Brasiliensis, 2(3):161-142, tab. 44-58 (BMN, BJB)

93 - DOMBROWSKI, L. T. D. - 1972 - Coleç5̄o de Pteridophyta do Paraná, no Instituto de Defesa do Patrimônio Nacional. Araucariana, Botånica, $2: 9$ (BPSO)

94 - DRUDE, O. - 1881 - Palmae I. Raphieae, Mauritieae, Cocoineae in MARTIUS, C. F. P. von, Flora Brasiliensis, 3 (2):253-460, tab. 61-106 (BMN, BJB)

95 - DRUDE, O. - 1882 - Palmae II. Geonomeae, Hyophorbeae, Iriarteae, Sabaleae in MARTIUS, C. F. P. von, Flora Brasiliensis, 3 (2):461-584, tab. 107-134. (BMN, BJB) 
96 - DUARTE, A. P. - 1965 - Contribuiçlo para o conhecimento da Flora do Estado da Guanabara. Arq. Jard. Bot., R. de Janeiro, XVIII:235 (B.JB)

97 - DUNGS, F. e G. F. J. PABST - 1967 - Orchidaceae Brasiliensis. Orquídea, 29 (3):127 (BPSO)

98-EICHLER, A. G. - 1864 - Magnoliaceae in MARTIUS, C. F. P. von, Flora Brasiliensis, 13(1):121-126, tab. 28-29 (BMN, BJB)

99 - EICHLER, A. G. - 1864 - Winteracese in MARTIUS, C. F. P. von, Flora Brasiliensis, 1):127-140. (BMN, BJB)

100 - EICHLER, A. G. - 1867 - Combretaceae in MARTIUS, C. F. P. von, Flora Brasiliensis, 14(2): 77-128, tab. 23-25. (BMN, BJB)

101 - EICHLER, A. G. - 1868 - Loranthaceae in MARTIUS, C. F. P. von, Flora Brasiliensis, 5(2):1-136, tab. 1-44 (BMN, BJB)

102 - EICHLER, A. G. - 1869 - Balanophoraceae in MARTIUS, C. F. P. von, Flora Brasiliensis, 4 (2):1-74, tab. 1-16 (BMN, BJB)

103 - EICHLER, A. G. - 1871 - Violaceae in MARTIUS, C. F. P. von, Flora Brasiliensis, 13 (1):345-396, tab. $69-80$ (BMN, BJB)

104 - EICHLER, A. G. - Bixaceae in MARTIUS, C. F. P. von, Flora Brasiliensis, 13 (1):421-516, tab. 86-103 (BMN, BJB)

105 - EICHLER, A. G. - Draseraceae in MARTIUS, C. F. P. von, Flora Brasiliensis, $14(2): 385-398$, tab. 90-91. (BMN, BJB)

106 - EICHLER, A. G. - Capparidaceae in MARTIUS, C. F. P. von, Flora Brasiliensis, 13 (1):237-292, tab. 54-65 (BMN, BJB)

107 - ENGLER, A. - 1871 - Escallonieae in MARTIUS, C. F. P. von, Flora Brasiliensis, 14 (2):129-148, tab. 36 (BMN, BJB)

108 - ENGLER, A. - 1871 - Cunoniaceae in MARTIUS, C. F. P. von, Flora Brasiliensis, 14 (2):149-172, tab. 37-39 (BMN, BJB)

109 - ENGLER, A. - 1872 - Olacineae in MARTIUS, C. F. P. von, Flora Brasiliensis, 12 (2):1-40, tab. $1-8$ (BMN, BJB)

110 - ENGLER, A. - 1872 - Icacineae in MARTIUS, C. F. P. von, Flora Brasiliensis, 12 (2):41-62, tab. 9-12, (BMN, BJB)

111 - ENGLER, A. - 1874 - Rutaceae in MARTIUS, C. F. P. von, Flora Brasiliensis, 12 (2):77-196, tab. 14-39 (BMN, B.JB)

112 - ENGLER, A, - 1878 - Araceae in MARTIUS, C. F. P. von, Flora Brasiliensis, 3 (2):25-224, tab. 2-52 (BMN, BJB)

113 - ENGLER, A. 1878 - Ochnaceae in MARTIUS, C. F. P. von, Flora Brasiliensis, 12 (2):297-366, tab. 62-77. (BMN, BJB)

114 - ENGLER, A. - 1888 - Guttiferae in MARTIUS, C. F. P. von, Flora Brasiliensis, 12 (1):381-474, tab. 79-108. (BMN, BJB) 
115 - ENGLER, A. - 1898 - Beiträge zur Kenntnis der Aracese. VIII. Revision der Gattung Anthurium Schott. Engl. Bot. JB. 25:352-476. (BJB)

116 - ENGLER, A. - 1905 - Araceae - Pothoideae in ENGLER, A. und K. PRANTL, das Pflan zereich, 21:1-333. (BMN)

117 - ENGLER, A. und K. KRA USE - 1908 - Additamentum and Araceaeas.Pothoideae; Araceas Monsteroideae: Aracese-Calloideae in ENGLER, A. und K. PRANTL, das Pflanzenreich, 37:1-160. (BMN)

118 - ENGLER, A. und K. KRAUSE - 1913 - Araceae-Philodendroideae-Philodendrineae in ENGLER, A. und K. PRANTL, Das Pflanzenreich, 60:1-143. (BMN)

119 - ENGLER, A. und K. KRAUSE - 1920 - Araceae-Colocasioideae; Addita, entum ad Aracese -Philodendroideas in ENGLER, A. und K. PRANTL, das Pflanzenreich, 71:1-139. (BMN)

120 - EPLING, C. e J. F. TOLEDO - 1943 - Labiadas in HOEHNE, F. C., Flora Brasilica, 48 (7):1-107, 42 tabs. (BPSO)

121 - ESENBECK, C. G. N. ab - 1842 - Cyperaceae in MARTIUS, C. F. P. von, Flora Brasiliensis. 2 (1):1-212, tab. 1-30. (BMN, BJB)

122 - ESENBECK. C. G. N. ab - 1847 - Acanthaceae in MARTIUS, C. F. P. von, Flora Brasiliensis, $9: 1-164$, tab. 1-31. (BMN, BJB)

123 - FOURNIER, E. - 1885 - Asclepiadaceae in MARTIUS, C. F. P. von, Flora Brasiliensis, 6 (4):189-332, tab. 50-98. (BMN, BJB)

124 - FRANCEY, P. - 1936 - Monographie du genre Cestrum L. De Candollea, 6:46-398. (BJB)

125 - FRANCEY, P. - 1938 - Monographie du genre Cestrum L. De Candollea, 7:1-132. (BJB)

126 - FRISTSCH. K. - 1901 - Beitrag zur Kenntnis der Gesneriaceen Flora Brasiliensis. Engl. Bot. JB., 29, Beibl. 65:5-23. (BMN)

127 - FRITSCH, K. - 1906 - Zwiter Beitrag zur Kenntnis der Gesneriaceen. Flora Brasiliensis. Engl. Bot. JB. 37:481-502. (BMN)

128 - GARDNER, G. - 1942 - Viagens no Brasil. Brasiliana, 5 (223) : 1-467.(BMN)

129 - GARDNER, G. - 1843 - Description of four New Genera of Plants from the Organ Mountains. Hook. Lond. Journ. Bot. 2:9-15. (BMN)

130 - GARDNER, G. - 1843 - Contribuitions towards a Flora of Brazil. Part II. Plants from the Organ Mountains. Hook. London. Journ. Bot. 2:339-355. (BMN)

131 - GAR DNER, G. 1843 - Notices of some Brazilian Fungi. Hook Lond. Journ. Bot. 2:9-15. (BMN)

132 - GARDNER, G. 1845 - Contribuitions towards a Flora Brazil, Being the distinctive characters of a century os nes species of Plants from the Organ Mountains. Hook. Lond Journ Bot. 4:97 - 137. (BMN)

133 - GARDNER, G. - 1846 - Contribuitions towards a Flora of Brazil being the distinctive characters of some new species of Compositae belonging to the tribe Vernoniaceac. Hook. Lond. Journ. Bot. 5:209-242. (BJB) 
134 - GIESEN, H. - 1938 - Triuridaceae in PRANTL, A. und K. PRANTL, Das Pflanzenreich, 104:1-84. (BMN)

135 - GRISEBACH, A. H. R. - 1858 - Malpighiaceae in MARTIUS, C. F. P. von, Flora Brasiliensis, $12(1): 1-108$, tab. 1-22. (BMN, BJB)

136 - GUIMARAES, E. F. e J. G. PEREIRA - 1965 - Typus do Herbário do Jardim Botånico do Rio de Janeiro, II. Arq. Jard. Bot. R. Janeiro, XVIII: 261. (BJB)

137 - GUiRKE, M. 1892 - Malvaceae II in MARTIUS, C. F. P. von, Flora Brasiliensis, 12(3):457-586, tab. 81-114. (BMN, BJB)

138 - HANDRO, O. e G. G. PABST - 1971 - Pleurothallis wacketti n. sp. Bradeia, 1 (7):45-48. (BPSO)

139 - HANSTEIN, J. - 1864 - Gesneriaceae in MARTIUS, C. F. P. von, Flora Brasiliensis, 8 (1):341-424, tab. 58-78. (BMN, BJB)

140 - HARMS, H. - Meliaceae americanae novae. Notizbl. Bot. Gart. Berl. 13(119):503. 1937. (BMN)

141 - HERZOG, T. - 1925 - Contribuiç̋̄es ao conhecimento da Flora Bryologica do Brasil. Arq. Bot. S. Paulo, 1(2):53. (BJB)

142 - HOEHNE, F. C. e J. G. KUHLMANN, Ultriculárias do Rio de Janeiro e seus arredores. Mem. Inst. Butantan, 1(1):5-21, 8 tab. (BJB)

143 - HOEHNE, F. C. - 1930 - Contribuiçōes para o conhecimento da Flora orchidologica Brasilica. Arq. Inst. Biol. Vegetal, 3:287-320. (BMN)

144 - HOEHNE, F. C. - 1938 - Cincoenta e uma novas espécies da flora do Brasil e outras descriçōes e ilustraçōes. Arq. Bot. Est. São Paulo, Nov. Sér., 1 (1):7-38, 45 tab. (BJB)

145 - HOEHNE, F. C. - 1939 - Dezoito espécies para a flora do Brasil e outras regiōes da América Meridional e Central, Arq. Bot. Est. Säo Paulo, Nov. Sér. 1(2):41. (BJB)

146 - HOEHNE, F. C. - 1940 - Orchidaceae. Flora Brasilica, 12, 1(1):1-254, 153 tab. (BPSO)

147 - HOEHNE, F. C. - 1941 - Leguminosas Papilionadas - Flora Brasilica, 25, 3 (3):3-100, 107 tab. (BPSO)

148 - HOEHNE, F. C. - 1941 - Leguminosas-Papilionadas. Flora Brasilica, 25, 3(4):1 39, 40 tab. (BPSO)

149 - HOEHNE, F, C. - 1942 - Orchidaceas. Flora Brasilica, 12, 6(5):1-218, 137 tab. (BPSO)

150 - HOEHNE, F. C. - 1945 - Orchidaceas. Flora Brasilica, 12, 2(8):1-389. (BPSO)

151 - HOEHNE, F. C. - 1952 - Espécies e variedades novas das Orquidaceas do Brasil. Arq. Bot. Est. S. Paulo, 2 (6):121-136. (BMN)

152 - HOEHNE, F. C. - 1953 - Orchidaceas. Flora Brasilica, 12, 7(10):1-397, 181 tab. (BPSO)

153 - HOOKER, J. D. - 1867 - Rosaceae in MARTIUS, C. F. P. von, Flora Brasiliensis, 14(2):1-74, tab. 1-22. (BMN, BJB) 
154 - HOOKER, W. J. and W. WILSON - 1844 - Enumeration of the Mosses and Hepaticae colleted in Brazil by George Gardner. Hook. Lond. Journ. Bot. 3:149-167. (BJB)

155 - HORNSCHUCH, C. F. - 1840 - Musci in MARTIUS, C. F. P. von, Flora Brasiliensis, $1(2): 1-96$, táb., 1-4 (BMN, BJB)

156 - HUBER, J. - 1896 - O "muricy" da Serra dos Orgăos. (Vochýsia goeldii n. sp.) B. Mus. Paraense, 2:382-385. (BPSO)

157 - JOHNSTON, J. M. - 1924 - On Some South American Proteacese. Contr. Gray Herb. Harv., N.S., 73:41. (BJB)

158 - JOLY, A. B. - 1970 - Conheça a vegetaçāo brasileira. Editora da Universidade de São Pauloe Polígono: Såo Paulo: 55. (BSO)

159 - KANITZ, A. - 1878 - Lobeliaceae in MARTIUS, C. F. P. von, Flora Brasiliensis, 6 (4):129-158, tab. 39-45. (BMN, BJB)

160 - KLATT, F. G. - 1871 - Irideae in MARTIUS, C. F. P. von Flora Brasiliensis, 3(1) $509-548$, tab. 64-71. (BMN, BJB)

161 - KOEHNE, B. A. E. - 1877 - Lythracese in MARTIUS, C. F. P. von, Flora Brasiliensis, 13(2):185-370, tab. 39-67. (BMN, BJB)

162 - KNUTH, R. - 1917 - Dioscoreacearum Americanae Novae. Notizbl. Bot. Grat. Berl., 7(65):185-222. (B.JB)

163 - KNUTH, R. - 1924 - Dioscoreaceae in ENGLER, A. und K. PRANTL, Das Pflanzenreich, 87:1-388. (BMN)

164 - KNUTH, R. - 1929 - Lecythidaceae in ENGLER, A. und K. PRANTL, Das Pflanzenreich, 105:67. (BMN)

165 - KNUTH, R. - 1930 - Oxalidaceae in ENGLER, A. und K. PRANTL, Das Pflanzenreich, 95:1-480. (BMN)

166 - KOERNICKE, F. - 1863 - Eriocaulaceae in MARTIUS, C. F. P. von, Flora Brasiliensis, 3 (1):273-502, tab. 38-60. (BMN, BJB)

167 - KOSTERMANS, A. J. G. J. 1936 - Revision of the Lauraceae I. Meded. Bot. Mus. Herb. Rijks-Univ. Utrecht, 31:719-757. (BMN)

168 - KRANZLIN, Fr. - 1912 - Cannaceae in ENGLER, A. und K. PRANTL, Das Pflanzenreich, 56:1-77. (BMN)

169 - KRANZLIN, Fr. - 1922 - Orchidaceae-Monandrae tribus Oncidiinae-Odontoglossae. Pars II. in ENGLER, A. und. K. PRANTL, Das Pflanzenreich, 80:1-344. (BMN)

170 - KRANZLIN, Fr. - 1923 - Orchidaceae-Monandrae-Pseudomonopodiales, in ENGLER, A. und K. PRANTL, Das Pflanzenreich, 83:1-66. (BMN)

171 - KRIEGER, W. - 1950 - Desmidiaceen aus der montanen Region Südost-Brasoçoems. Ber. Dt. Bot. Ges, 63(2):36-43. (BJB)

172 - KRONFELD, M. - 1894 - Thyphaceae in MARTIUS, C. F. P. von, Flora Brasiliensis, 3(3):635-642, tab. 115. (BMN, BJB) 
173 - KUHLMANN. J. G. - 1946 - Uma nova Bignonacea da Serra dos Orgăos. Rodriguésia, 10 (2):7-8, 1 tab.

174 - KUKENTHAL, G. - 1909 - Cyperaceae-Caricoidese in ENGLER, A. und K. PRANTL, Das Pflanzenreich, 38:1-824 (BMN)

175 - KUKENTHAL, G. - 1935-1936 - Cyperus in ENGLER, A. und K. PRANTL, Das Pflanzenreich, 101(4):490. (BMN)

176 - LINDLEY, J. - 1843 - Character of four news species of Orchidaceae from Mr. Gardiner's First Organ Mountain Collection. Hook. Lond. Journ. Bot. 2:661-663. (BMN)

177 - LOEF GREN, A. - 1915 - O gênero Rhipsalis. A rq. Jard. Bot. R. Janeiro, L.63-104, 25 tabs. (BJB)

178 - LOEFGREN, A. - 1918 - Novas Contribuições para as Cactáceas Brasileiras; sôbre os gêneros Zygocactus e Schlumbegera. Arq. Jard. Bot. R. Janeiro, 2:1-32, 4 tabs. (BJB)

179 - MARCHAL, E. - 1878 - Hederaceae in MARTIUS, C. F. P. von, Flora Brasiliensis, 11 (1):221-258, tab. 66-71. (BMN, BJB)

180 - MAGNANI NI, A. e M. T. JORGE - 1969 - Situacăo dos Parques Nacionais no Brasil. B. Inf. Fund. Bras. Conserv. Nat. 4:52-53. (BPSO)

181 - MARTIUS, C. F. P. von - Icones Plantarum Cryptogamicarum. 138 pp., 76 tabs. (BMN)

182 - MARTIUS, C. F. P. von - 1841 - Tabulae Physiogonomicae Brasiliae - 6. Silva primitiva in Serra dos Orgăos, Prov. Rio de Janeiro in MARTIUS, C. F. P. von, Flora Brasiliensis, 1(1):IX-XVII, tab, 6. (BMN, BJB)

183 - MARTIUS, C. F. P. von - 1841 - Anonaceae in MARTIUS, C. F. P. von, Flora Brasiliensis, 13 (1):1-48, tab. 1-14. (BMN, BJB)

184 - MARTIUS, C. F. P. von - 1855 - Tabulae Physiogonomicae 44. Cultura coffeae in praedio inter oppidum Magé et Montes Serra dos Órgāos in MARTIUS, C. F. P. von, Flora Brasiliensis, 1(1), tab. 44. (BMN, BJB)

185 - MARTIUS, C. F. P. von - 1869 - Tabulae Physiogonomicae 59. Silva Montium Serra dos Orgäos declivia obrumbrans, in prov. Rio de Janeiro in MARTIUS, C. F. P. von, Flora Brasiliensis, 1(1), tab. 59. (BMN, BJB)

186 - MASTERS, M. I. - 1872 - Passifloracese in MARTIUS, C. F. P. von, Flora Brasiliensis, 13(1): 529-628, tab. 106-128. (BMN, BJB)

187 - MASTERS, M. I. - 18875 - Aristolochiaceae in MARTIUS, C. F. P. von, Flora Brasiliensis, 4 (2):77-112, tab. 17-26. (BMN, BJB)

188 - MEISNER, C. F. - 1855 - Polygalacese in MARTIUS, C. F. P. von, Flora Brasiliensis, 5 (1):1-60, tab. 1-27. (BMN, BJB)

189 - MEISNER, C. F. - 1855 - Proteaceae in MARTIUS, C. F. P. von, Flora Brasiliensis, 5(1):73-100, tab. 31-36. (BMN, BJB)

190 - MEISNER, C. F. - 1863 - Ericaceae in MARTIUS, C. F. P. von, Flora Brasiliensis, 7:119-174, tab. 48-66 (BMN, BJB) 
191 - MEISSNER, C. F. - 1866 - Lauraceae in MARTIUS, C. F. P. von, Flora Brasiliensis, 5 (2):137-296, 301-302, tab, 45-107. (BMN, BJB)

192 - MELLO Filho, L. E. de - 1948 - Importancia biogeográfica de recentes modificaçöes pro' postas ao Código Florestal. Assoc. Geogr. Brasileiros, 1:3-5. (BPSO)

193 - MELLO Filho, L. E. de - 1960 - Consideraç̋̄es sobre Rauvolfia sellow. Muell. Arg. B. Mus. Nac., Nova Sér., Botânica, 26:1-9, 3 tab. (BMN)

194 - MELLO Filho, L.E. e J. C. C. BARCIA - 1976 - Germinaçăo de Strychos brasiliensis (Spreng.) Mart. em condiçōes experimentais. Anais da Sociedade Botânica do Brasil. 259 Congr. Nac. de Botånica, Mossoró, RN, 1974:247-258, 14 figs. (BPSO)

195 - MEZ C. - 1892 - Bromeliaceae II in MARTIUS, C. F. P. von, Flora Brasiliensis, 3(3): 279-430, tab. 63-80. (BMN, BJB)

196 - MEZ, C. - 1894 - Bromeliaceae III in MARTIUS, C. F. P. von, Flora Brasiliensis, 3(3):429-634, tab. 81-114. (BMN, BJB)

197 - MEZ, C. 1902 - Myrsinaceae in ENGLER, A. K. PRANTL, Das Pflanzenreich, 9:1437. (BMN)

198 - MIERS, J. - 1846 - Contribuitions to the Botany of South America. Hook. Lond Journ. Bot., 5:144-190. (BJB)

199 - MICHELI, M. - 1875 - Onagraceae - in MARTIUS, C . F. P. von, Flora Brasiliensis, 13 (2):145-180, tab. 28-38. (BMN, BJB)

200 - MIQUEL, F. A. G. - 1853 - Urticineae in MARTIUS, C. F. P. von, Flora Brasiliensis, 4 (1):77-218, tab. 25-70. (BMN, BJB)

201 - MIQUEL, F. A. G. - 1856 - Simplocaceae in MARTIUS, C. F. P. von, Flora Brasiliensis. 7:21-36, tab. 8-14. (BMN, BJB)

202 - MIQUEL, F. A. G. - 1856 - Myrsinaceae in MARTIUS, C. F. P. von, Flora Brasiliensis. 10:269-324, tab. 23-59. (BMN, BJB)

203 - MOREIRA, A. X. - 1969 - Catálogo de pólens do Estado da Guanabara e arredores. Museu Nacional, $R$ io de Janeiro: 1-49. (BMN)

204 - MORS, W.B. - 1952 - Investigaçōes químicas sobre Líquens Brasileiros. Estudo das Usnest da Serra dos Orgắos. B. Inst. Quím. Agrícola, 29:7-23. (BPSO)

205 - MULLER, C. - 1849 - Synopsis Muscorum Frondosorum I. Berlim, 812 pp. (BJB)

206 - MULLER, C. - 1852 - Synopsis Muscorum Frondosorum II. Berlin. 172 pp. (BJB)

207 - MULLER, C. - 1900 - Symbolae ad Bryologiam Brasiliae et Regionum Vicinarum. Hedwigia. 39:235-289. (BJB)

208 - MULLER, C. - 1901 - Symbolae ad Bryologiam Brasiliae et Regionum Vicinarum. Hedwigia 40:55-99. (BJB)

209 - MULLER, C. A. - 1885 - Valerianaceae in MAR TIUS, C. F. P. von, Flora Brasiliensis, 6 (4):339-350, tab. 100-102. (BMN, B.JB) 
210 - MULLER-ARG., J. - 1860 - Apocynacese in MARTIUS, C. F. P. von, Flora Brasiliensis, 6 (1):1-180, tab. 1-53. (BMN, BJB)

211 - MULLER-ARG., J. - 1873 - Euphorbiaceae I. Phyllantheae et Crotoneae in MARTIUS, C. F. P. von, Flora Brasiliensis, 11(2):1-292, tab. 1-42. (BMN, BJB)

212 - MULLER.ARG., J. - 1874 - Euphotblaceae II. Acalypheae, Hippomaneae, Dalechampiae, Euphorbiae in MARTIUS, C. F. P. von, Flora Brasiliensis, 11 (2):293-721, tab. 43-104. (BMN, BJB)

213-MULLER-ARG., J. - 1881 - Rubiaceae I. Retiniphylleae, Guettardeae, Chiococeeae, Ixoreae, Coussareae, Psycotrieae in MARTIUS, C. F. P. von, Flora Brasiliensis, 6 (5):1-470, tab. 1-67. (MBN, BJB)

214 - MULLER, J. - 1891 - Lichenes Schenckiani. Hecwigia, 30(5):219-243. (BJB)

215 - MUNZ, P.A. - 1947. Onagraceae in HOEHNE, F. C. - Flora Brasilica, 41, 1 (9): 1-62, 51 tab. (BMN)

216 - NESSEL, H. - 1927 - As Lycopodiaceas do Brasil. Arq. Bot. Est. S. Paulo, 1(4):359-447, 44 tab.

217 - NESSEL. H. - 1939 - Die Barlappgewachse (Lycopodiaceae). Jena. 404 pp. (BJB)

218 - NESSEL, H. - 1955 - Lycopodiaceae in HOEHNE, F. C. - Flora Brasilica, 2,2(11):1-131 (BPSO)

219 - NIDENSEN, Fr. - 1928 - Malpighiacese in ENGLER, A. und K. PRANTEL, Das Pflanzenreich, 91:1-246. (BMN)

220 - NIDENSEN, Fr. - 1928 - Malpighiaceae in ENGLER, A. und K. PRANTEL, Das Pflanzenreich, 93: 247-572. (BMN)

221 - OCCHIONI, P. - Contribuição ao Estudo Botânico da "Casca-d'Anta" - Drymys brasiliensis Miers. Arq. Jard. Bot. R. Janeiro, 7:135-155, 13 figs. (BJB)

222 - OCCHIONI, P. - 1947 - Nova Espécies de "Canellacese". Arq. Jard. Bot. R. Janeiro, 7:157-163, 1 tab. (B.JB)

223 - OCCHIONI, P. - 1948 - Nota sobre a Biologia das Canelacees Brasileiras. Arq. Jard. Bot. R. Janeiro, 8: 275-279. (BJB)

224 - OCCHIONI, P. - 1952 - Contribuição ao estudo do gênero Oxypetalum. Com especial referência ds Spp. da Serra do Itatiaia e Serra dos Orglios. Arq. Jard. Bot. R. Janeiro, 14:43-210. (BJB)

225 - PABST, G. F. J. - 1951 - Notícias Orquidológicas. I. Rodriguesia, Rio de Janeiro, 14:26-55. 1 tab. (BJB)

226 - PABST, G. F. J. - 1956 - Additamenta ad Orchilogiam Brasiliensen. II. Arq. Jard. Bot. R. Janeiro XXIV:1-27, 7 tab. (BJB)

227 - PABST, G. F. J. - 1956 - Additamenta ad Orchilogiam Brasiliensen. III. Rodriguésia, R. Janeiro, 18 e 19 (30 e 31): 29. (BJB)

228 - PABST. G. F. J. - 1967 - Notícias Orquidológicas. X. Orquídea, 29 (3):114, 1 tab. 
229 - PABST, G. F. J. - 1972 - Additamenta ad Orchideologiam Brasiliensen. X Bradea, 1 (19):171. (BPSO)

230 - PAX. F. - 1910 - Euphorbiaceae-Adrianese in ENGLER, A. und K. PRANTL, Das Pflan zenreich, 44:61. (BMN)

231 - PAX, F. und K. HOFFMANN - 1912 - Euphorbiacese-Gelonieae; Euphorbiacese-Hippoma neae in ENGLER, A. und K. PRANTL, Das Pflanzenreich, 52:1-319. (BMN)

232 - PAX, F. und K. HOFF MANN - 1914 - Euphorbiacese - Acalypheae - Mercurialinae in ENGLER, A. und K. PRANTL, Das Pflanzenreich, 63:233. (BMN)

233 - PAX, und K. HOFFMANN - 1919 - Euphorblaceae - Acalypheae - Plukenetunae in ENGLER, A. und K. PRANTL, Das Pflanzenreich, 68:49. (BMN)

234 - PAX, F. und K. HOFFMANN - 1924 - Euphorbiaceae in ENGLER, A. und K. PRANTL. Das Pflanzenreich, 85:231. (BMN)

235 - PEREIRA, E. - 1957 - A Flora do Itatiaia. I. - Saxifragaceae. Rodriguésia, 20 (32): 242-243. (BMN)

236 - PEREIRA, E. - 1971 - Species nova in Brasilia Bromeliacearum. Rodriguésia. 26(38):113-117, 8 tab. (BJB)

237 - PEREIRA, J. P. - 1963 - Os tipos das plantas vasculares do Herbário do Museu Nacional. II. Myrsinaceae. B. Mus. Nac., Nova Ser., Botânica, 29:18. (BMN)

238 - PEREIRA, J. F., M. C. VALENTE e F. M. M. R. de ALENCASTRO - 1971 - Contribuie 50 ao Estudo das Asclepiadaceae Brasileiras. V. Estudo Taxinðmico a Anatômico de Oxypetalum Banksii Roem. et Schult. Rodriguésia, 26 (38): 261-276, 5 tab. (BJB)

239 - PEREIRA, J. F. e N. M. F. SILVA - 1972 - Estudos em Asclepiadaceae I. Novos Sinônimos. Bradea, 1 (14):133 (BPSO)

240 - PERKINS, J. und E. GILG - 1901 - Monimiaceae in ENGLER, A. und K. PRANTL, D\$s Pflanzenreich, 4:1-222. (BMN)

241 - PETERSEN, O. G. - 1890 - Zingieberaceae in MAR TIUS, C. F. P. von, Flora Brasiliensis, ${ }^{3}$ (3):29-62, tab. 9-14. (BMN, BJB)

242 - PICKEL, B. - 1937 - Catálogo do Herbário da Escola Superior de Agricultura em Taper3 (PE). B. Mus Nac, 13 (1-2):63.132. (BMN)

243 - PILGER, R. - 1923 - Plantae Lutzelburgianae Brasilienses. I. Notizbl. Bot. Gart. Berl., 8 (76):425-451. (BJB)

244 - PILGER, R. - 1937 - Plantaginaceae in ENGLER, A. und K. PRANTL, Das Pflanzenreich. 102: 1-466. (BMN)

245 - PINTO, G. S. - 1949 - Primeira Revista do Parque Nacional da Serra dos OrgJ̃os. Serviç Florestal, Ministério da Agricultura. 76 pp. (BPSO)

246 - POHL, J. E. - 1951 - Viagem no Interior do Brasil. I. Instituto Nacional do Livro. 400 pp. (BMN)

247 - PROGEL, A. - 1865 - Gentianacese in MARTIUS, C. F. P. von, Flora Brasiliensis, 6 (1):197-246, tab. 55-66. (BMN, BJB) 
248 - PROGEL, A. - 1868 - Loganiaceae in MARTIUS, C. F. P. von, Flora Brasiliensis, 6 (1):249-290, tab. 67-82. (BMN, BJB)

249 - PROGEL, A. - 1877 - Oxalidaceae in MARTIUS, C. F. P. von, Flora Brasiliensis, 12 (2):473-520, tab. 102-116. (BMN, BJB)

250 - RADLKOFER, L. - 1892 - Sapindacese I. in MARTIUS, C. F. P. von, Flora Brasiliensis, 13 (3):225-346, tab. 58-80. (BMN, BJB)

251 - RADLKOFER, L. - 1897 - Sapindaceae II., in MARTIUS, C. F. P. von, Flora Brasiliensis. 3(3):345-464, tab. 81-89. (BMN, BJB)

252 - RADLKOFER, L - 1900 - Sapindaceae III. in MARTIUS, C. F. P. von, Flora Brasiliensis, 13 (3):465-658, tab. 100-123. (BMN, BJB)

253 - RADLKOFER, L. - 1931-1934 - Sapindaceae in ENGLER, A. und K. PRANTL, Das Pflanzenreich, 98 (1):1-321. (BMN)

254 - RADLKOFER, L. - 1931-1934 - Sapindaceae in ENGLER, A. und K. PRANTL, Das Pflanzenreich, 98 (2):321-640. (BMN)

255 - RADLKOFER, L. - 1931-1934 - Sapindaceae in ENGLER, A. und K. PRANTL, Das Pflanzenreich, 98 (5):961-114. (BMN)

256 - REHM, H. - 1900 - Beitrage zur Pilsflora von Sudamerica. VIII. - Discomycetes. Hedwgia, 39:211. (BJB)

257 - REICHARDT, H. J. - 1878 - Hypericaceae in MARTIUS, C. F. P. von, Flora Brasiliensis, 12 (1):181-212, tab. 33-39. (BMN, BJB)

258 - REISSEK, S. - 1891 - Celastraceae in MARTIUS, C. F. P. von, Flora Brasiliensis, 11 (1):1-36, tab. 1-10. (BMN, BJB)

259 - REISSEK, S. - 1861 - Ilicineae in MARTIUS, C. F. P. von, Flora Brasiliensis, 11 (1):37-80, tab. 11-23. (BMN, BJB)

260 - REISSEK, S. -1861 - Rhamneae in MARTIUS, C. F. P. von, Flora Brasiliensis, $11(1): 81-126$, tab. 24-41. (BMN, BJB)

261 - RIZZINI, C. T. - 1946 - Aliquit Novi Acanthacearum. R. Bras. Biol., 6(4):521-525, 21 figs. (BMN)

262 - RIZZINI, C. T. - 1948 - Disquisito Circa Acanthacearum Aliquot Genera Brasiliensis. A rq. Jard. Bot. R. Janeiro, 8:295-372, 8 tabs. (BJB)

263-RIZZINI, C. T. - 1950 - De Plantis Brasiliensis Nonnullis. Dusenia, 1 (5) 289-296, 1 tab. (BMN)

264-RIZZINI, C. T. - 1950 - Struthanthi Brasiliae Eiusque Vicinorum. R. Bras, Biol., 10 (4):393-408. (BMN)

265 - RIZZINI, C. T. - 1952 - On a new Brazilian Hemelichen - Arq. Jard. Bot. R. Janeiro. $12: 137-144,1$ tab. (BJB)

266 - RIZZINI, C. T. - 1952 - Dichapetalaceae Brasiliensis. R. Bras. Biol., 12 (1) $97-108$. (BMN) 
267 - RIZZINI, C. T. - 1952 - Species Organenses Generis Lichenum Usneae (Omnes Acidium Usnicum Praebentes). R. Bras. Biol., 12 (4):337-348. (BMN)

268 - RIZZINI, C. T. - 1953-1954 - Flora Organenis. Lista preliminar dos Cormophyta da Serra dos Orgäos. Arq. Jard. Bot. R. Janeiro, 13:1 15-243, 17 tabs. (BJB)

269 - RIZZINI, C. T. - 1956 - Flora Organensis. Lichenes. R. Bras. Biol., $16(4): 387-402$. (BJB)

270 - RIZZINI, C. T. - Pars specialis prodomi monographiae Loranthacearum Brasiliae Terrarum que Finitimarum. Rodriguesia, 18 e 19 (30 e 31):87-234, 29 tabs. (BJB)

271 - RIZZINI, C. T. e M. M. PINTO - 1964 - Areas clímaco-vegetacionais do Brasil, segundo os métodos de Thornthwaite e de Hohr. R. Bras. Geogr., 26 (4):536. (BMN)

272 - RUHLAND, W. 1903 - Eriocaulaceae in ENGLER, A. und K. PRANTL, Das Pflanzenreich, 13:1-294 (BMN)

273 - SAMBO, M. C. - 1940 - Licheni del Brasile. Annali Bot., 22 (1):19-41. (BJB)

274 - SANDWITH, N. Y. - 1930 - Contributions to the Flora of Tropical America. Kew Bull., $1: 210-215$. (BJB)

275 - SANTESSON, R. - 1942 - The South American Cladinae. Ark. f. Bot. Band 30A (10):1-27. (BMN)

276 - SANTESSON, R. - 1952 - Follicolus Lichens I. Symb. Bot. Upsal. XII:1-590. (BJB)

277 - SANTOS, E. A. A. dos - 1963 - Os tipos das plantas vasculares do Herbário do Museu Nacional II. B. Mus. Nac. Nova Sér., Botảnica, 29:13. (BMN)

278 - SANTOS, E. A. A. dos - 1963 - Os tipos das plantas vasculares do Herbário do Museu Nacional II. Sterculiaceae B. Mus Nac., Nova Sér., Botànica, 29:13 (BMN)

279 - SANTOS, E. A. A. dos - Droseraceae do Rio de Janeiro, Brasil. B. Mus. Nac. Nova Sér. Botånica, 35:4,1 tab. (BMN)

280 - SCHAUER, J. C. - 1851 - Verbenaceae in MARTIUS, C. F. P. von, Flora Brasiliensis. 9:169-308, tab. 32-50. (BMN, BJB)

281 - SCHEINVAR, L - 1963 - Os tipos das plantas vasculares do Herbário do Museu Nacional II. Cucurbitaceae B. Mus, Nac, Nova Sér., Botånica 29-21. (BMN)

282 - SCHRENCK, H. - 1892 - Brasilianische Pteridophyten. Hedwigia, 35:141-172. (BMN)

283 - SCHENK, A. - 1855 - Alstromerieae in MARTIUS, C. F. P. von, Flora Brasiliensis, 3 (1): $165-180$, tab 20 e 21. (BMN, BJB)

284 - SCHLECHTER, R. - 1920 - Studium zur Klarung der Gattung Rodriguésia Ruiz et PavFedde Reppert., 16:425-430. (BJB)

285 - SCHLECHTER, R. - 1921 - Orchidaceae novae et criticae. Decas LXX. Fedde Repertu $17: 267 \cdot 272$. (BJB)

286 - SCHLECHTER, R. e F. C. HOEHNE. Contribuiçōes ao conhecimento das Orchidaceas do Brasil. III. Arq. Bot. Est. S. Paulo, 1 (3):165-298, 26 tabs. (BJB) 
287 - SCHMIDT, J. A. - 1858 - Labiatae in MARTIUS, C. F. P. von, Flora Brasiliensis, 8 (1):65-204, tab. $14-38$ (BMN, BJB)

288 - SCHMIDT, J. A. - 1862 - Scrophularinae in MARTIUS, C. F. P. von, Flora Brasiliensis, 8 (1):229-330, tab. 39-57. (BMN, BJB)

289 - SCHMIDT, J. A. - 1872 - Nyctagineae in MARTIUS, C. F. P. von, Flora Brasiliensis, 14 (2):334-374. (BMN, BJB)

290 - SCHMIDT, J. A. - 1878 - Plantaginacese in MARTIUS, C. F. P. von, Flora Brasiliensis, 6 (4):169-176, tab. 47. (BMN, BJB)

291 - SCHUMANN, C. - 1886 - Bombacaceae in MARTIUS, C. F. P. von, Flora Brasiliensis, 12 (3):201-250, tab. 40-50. (BMN, BJB)

292 - SCHUMANN, D. - 1888 - Rublaceae Ila. Paederiae, Spermacoceae, Stellatae in MARTIUS, C. F. P. von, Flora Brasiliensis, 6 (6):1-124, tab. 68-93. (BMN, BJB)

293 - SCHUMANN, C. - 1889 - Rubiaceae IIb. Naucleeae, Henriquezieae, Cinchoneae, Rondeletieae, Condamineae, Hedytideae, Mussaendrae, Catesbaeeae, Hamelieae, Gardenieae in MARTIUS, C. F. P. von, Flora Brasiliensis, 6 (6):125-442, tab. 94-151. (BMN, BJB)

294 - SCHUMANN, C. - 1891 - Malvacese I. in MARTIUS, C. F. P. von, Flora Brasiliensis, 12 (3):251-456, tab. 51-80. (BMN, BJB)

295 - SCHUMANN, C. - 1891 - Cactaceae in MARTIUS, C. F. P. von, Flora Brasiliensis, 4 (2):185-323, tab. 39-63. (BMN, BJB)

296 - SCHUMANN, C. - 1894 - Triuridacese in MARTIUS, C. F. P. von, Flora Brasiliensis, 3 (3): $643-668$, tab. 116-117 (BMN, BJB)

297 - SCHUMANN, K. - 1902 - Marantaceae in ENGLER, A. und K. PRANTL, Das Pflanzenreich, $11: 1-184$ (BMN)

298 - SEUBERT, M. - 1847 - Burmaniaceae in MARTIUS, C. F. P. von, Flora Brasiliensis, 3 (1):53-60, tab. 7 fig. 2 a 4 . (BMN, BJB)

299 - SEUBERT, M. - 1847 - Vellosieae in MARTIUS, C. F. P. von, Flora Brasiliensis, 3 (1):65-84, tab. 8-10. (BMN, BJB)

300 - SEUBERT, M. - 1847 - Amaryllideae in MARTIUS, C. F. P. von, Flora Brasiliensis, 3 (1):141-164. (BMN, BJB)

301 - SEUBERT, M. - 1855 - Commelinaceae in MARTIUS, C. F. P. von, Flora Brasiliensis, 3 (1):243-270, tab. 32-37. (BMN, BJB)

302 - SEUBERT, M. - 1875 - Amarantacese in MARTIUS, C. F. P. von, Flora Brasiliensis, 5 (1):161-252, tab. 50-75. (BMN, BJB)

303 - SLEUMER, H. - 1937 - Ericaceae Americanae novae vel minus cognitae III. Notizbl. Bot. Gart. BerL., 13 (117):208. (BMN)

SMITH, L. B. - 1943 - Bromelias novas ou interessantes do Brasil. II. Arq. Bot. Est. S. Paulo, Nova Sér., 1 (5):101-122, 35 tabs. (BJB) 
306 - SMITH, L. B. - 1950 - Bromeliácees notáveis do Herbário do Jardim Botánico do Rio de Janeiro. Arq. Jard. Bot. R. Janeiro, 10:147. (BJB)

307 - SMITH, L. B. - 1952 - Bromeliáceas novas ou interessantes do Brasil. V. Arq. Bot. Est. S. Paulo, 2 (6):196. (BMN)

308 - SMITH, L. B. - 1957 - Xiridáceas Brasileiras do Herbário do Museu Nacional. B. Mus. NaC. Nova Sér., Botânica, 17:1-19. (BMN)

309 - SNETHLAGE, E. H. - 1923 - Nun Arten der Gattung Cecropia nebst Beitragen zu ihref Synonymik. Notizbl. Bot. Gart. Berlin, 8 (75):357-369. (BJB)

310 - SOUZA, A. B. de - 1971 - Catálogo da Carpoteca do Jardim Botânico. Parte I. Rodriguesia. 26 (38): 339-365. (BJB)

311 - SPRING, A. F. - 1840 - Lycopodineae in MARTIUS, C. F. P. von, Flora Brasiliensis. $1(2): 105-134$, tab. 5-8. (BMN, BJB)

312 - SGANDLEY, P. C. - 1930 - Studies of American Plants. IV. - Publs. Field. Mus. Nat. Hist. Bot. Ser., 8 (2):133-236. (BJB)

313 - STANDLEY, P. C. - 1936 - Studies of American Plants. VI. Publs. Field Mus. Nat. Hist Ser., 11 (5):145-276. (BJB)

314 - STAFLEU. F. A. - A monograph of the Vochysiaceae - I. Sa/vertia and Vochysia. Maded Bot. Mus. Herb. Rijks-Univ. Utrecht, 95:398. (BMN)

315 - STENDTNER, O. - 1846 - Solanaceae in MARTIUS, C. F. P. von, Flora Brasiliensis. $10: 5-200$, tab. 1-19. (BMN, BJB)

316 - STENDTNER, O. - 1846 - Crestineae in MARTIUS, C. F. P. von, Flora Brasiliensis. 10:201-208. (BMN, BJB)

317 - STEPHANI, F. - 1906 - Species Hepaticarum. (Species Hepaticarum II; Acrogynae). Bul' Herb. Boisser, Genève, 2(2):174. (BJB)

318 - STEPHANI, F. - 1906 - Species Hapaticarum (Species Hepaticarum II: Acrogynae). BulHerb. Boisser, Genève, 2, 5 (2):181. (BJB)

319 - STEPHANI, F. - 1906 - Species Hepaticarum (Species Hepaticarum II. Acrogynae). Bu Herb. Boisser, Genève, 2 (8) 677 . (BJB)

320 - STRANG, H. E. H. P. VELLOSO - 1969 - Parques Nacionais e Reservas Equivalentes Brasil. Ministério da Agricultura, 48 pp. (BPSO)

321 - STURM, J. G. - 1859 - Osmundaceae in MARTIUS, C. F. P. von, Flora Brasilienth $1(2): 161-166$, tab. 12 (BMN, BJB)

322 - STURM, J. G. - 1859 - Schizaeacees in MARTIUS, C. F. P. von, FIora Brasiliensish. (2):167-216, tab. 13-16. (BMN, BJB)

323 - STURM, J. G. - 1859 - Hymenophyllacese in MARTIUS, C. F. P. von, Flora Brasiliensis. (2):239-302, tab. 18 e 19. (BMN, BJB)

324 - SWART, J. J. - Novitates Burseracearum. Meded. Bot. Mus. Herb. Rijks-Univ. Utrecht89:189-210. (BMN) 
325 - TAUBERT, P. - 1893 - Revision der Gattung Griselinia. Engl. Bot. JB., 16:392. (BJB)

326 - TEIXEIRA, L. - 1959 - Basellaceae da cidade do Rio de Janeira. Rodriguesia, 21 e 22 l33 e 34):317-324, 3 tabs. (BJB)

327 - TOLEDO, J. F. - 1944 - Estudos sobre algumas palmeiras do Brasil I. Um novo Gênero da tribu Cocoeae. Arq. Bot. Est. S. Paulo, Nova Sér., 2 (1):3-9, 3 tabs. (BMN)

328 - TRA VASSOS, O. P. - Notas sobre "Typus" do Herbário do Museu Nacional - Begoniaceae. B. Mus. Nac. Nova Sér., Botânica, 25, 5 pags. (BMN)

329 - TRA VASSOS, O. P. - 1961 - Bignoniaceae in CASTELLANOS, A., Os Tipos das Plantas Vasculares do Herbário do Museu Nacional I. B. Mus. Nac., Nova Sêr., Botânica, 28:17. (BMN)

330 - TRA VASSOS, O. P. - 1965 - Typus do Herbário do Jardim Botánico do Rio de Janeiro. Arq. Jard. Bot. R. Janeiro, XVIII252. (BJB)

301 - TULASNE, L. R. - 1855 - Podostemacese in MARTIUS, C. F. P. von, Flora Brasiliensis, 4 (1):229-274, tab. 73-76. (BMN, BJB)

332 - TULASNE, L. R. - 1857 - Monimiaceae in MARTIUS, C. F. P. von, Flora Brasiliensis, 4 (1):289-328, tab. $82-856$.

333 - URBAN, I. - 1877 - Lineae in MARTIUS, C. F. P. von, Flora Brasiliensis, 12 (2):455-472, tab. 97-101. (BMN, BJB)

334 - URBAN, I. - 1879 - Umbelliferae in MARTIUS, C. F. P. von, Flora Brasiliensis, 11(1):261-354, tab. 72-91. (BMN, BJB)

335 - URBAN, I. de - 1897 - Plantae novae imprimis Glaziovianae I. Engl. Bot. JB, 23, Beibl. 57:1-16. (BMN)

336 - VATTIMO, I. de - 1956 - Lauraceae do Itatiaia. Rodriguésia, 18 e 19 (30 e 31):39-72. (BJB) 337 - VATTIMO, I. de - 1957 - A Flora do Itatiaia - I. Menispermaceae. Rodriguesia, 20 (32):43-44. (BJB)

338 - VATTIMO. I. de - 1957 - A Flora do Itatiaia - 1. Winteraceae. Rodriguesia, 20 (32):43-44. (BJB)

339 - VATTIMO, I. de - 1957 - A Flora do Itatiaia - I. Annonaceae. Rodriguesia, 20 (32):45-50, 1 tab. (BJB)

340 - VATTIMO, I. de - 1957 - A Flora do Itatiaia - I. Myristicaceae. Rodriguesia, 20(32):53-55. (BJB)

341 - VATTIMO, I. de - 1957 - A Flora do Itatiaia - I. Monimiaceae. Rodriguésia, 20 (32):56-61. (BJB)

342 - VELLOSO, H. P. - 1945 - As Comunidades e as Estações Botânicas de Teresópolis, Estado do R io de Janeiro. B. Mus. Nac., Nova Sér., Botânica 3:1-95, 36 tabs. (BMN)

343 - VELLOSO, H. P. e H. E. STRANG - 1970 - Alguns Aspectos Fisionômicos da Vegetação do Brasil. Mem. Inst. Oswaldo Cruz, 68 (1) $: 46-47$ (BPSO) 
344 - VIDAL, W. N. - 1963 - Os tipos das plantas vasculares do Herbário do Museu Nacional - II. Meliaceae. B. Mus., Nac., Nova Sér., Botånica, 29:10. (BMN)

345 - VIEIRA, A. - 1939 - Therezópolis, Rio de Janeiro, Jornal do Commércio, 25:35 (BPSO)

346 - WANGERIN, W. - 1910 - Cornaceae in ENGLER, A. und K. PRANTL, Das Pflanzenreich. 41:1-110. (BMN)

347 - WAR MING, E. - 1875 - Vochysiacese in MARTIUS, C. F. P. von, Flora Brasiliensis, 13 (2):17-114, tab. 2-21. (BMN, BJB)

348 - WITTIMACK, L. - 1878 - Marcgraviaceae in MARTIUS, C. F. P. von, Flora Brasiliensis, 12 (1):213-258, tab. 40-51. (BMN, BJB)

349 - WOLFF, H. - 1913 - Umbelliferae Saniculodeae in ENGLER, A. Und K. PRANTL, D\$ Pflanzenreich, 61:1-305. (BMN)

350 - WOODSON, R. E. - 1935 - Studies in the Apocynaceae - IV. The American Genera of Echitoideae. An Missouri Bot. Gard., 22:153-306. (BMN)

351 - ZAHZBRUCKNER, S. - 1902 - Studien uber brasilianische Flechten. Stizgb. d. Kais. Akad. Wiss. Wien. Nath-nature, CL, 111 (1):1-76, 2 tabs.

\section{ÍNDICE POR ASSUNTO}

(Os números referem-se ao indice dos autores)

Acalyphese: 212, 232, 233.

Acanthaceae: 122.

Acanthacearum: 261, 262.

Acrogiinae: 317, 318, 319.

Adrianae: 230.

Agrostideae: 91 .

Algas: 32.

Alismataceae: 18.

Alstroemerieae: 283.

Amaranthaceae: 301 .

Amaryllidese: 300.

Amaryllidaceae: 18.

Ampelideae: 13.

Anonaceae: 183.

Annonaceae: 339.

Anthemideae: 17.

Anthurium: 115.

Apocynacese: 210, 350.

Araceae: 18, 112, 115, 116, 117, 118, 119.

Aristolochiaceae: 187.

Arundinaceae: 91.

Asclepiadaceae: 6, 123, 238, 239.

Asteroideae: 16.

Avenaceee: 91.

Balanophoraceae: 102.

Bambusa: 92.

\author{
Barrintoineae: 30 \\ Basselaceae: 326. \\ Begonias: 41, 51. \\ Begoniaceae: $39,84,85,328$. \\ Bertolonieae: 70. \\ Bignoniacea: 173. \\ Bignoniaceae: 56, 57, 329. \\ Bixaceae: 104. \\ Blakeae: 72. \\ Bombacaceae: 291. \\ Bromeliaceae: 195, 196. \\ Bromeliacearum: 236. \\ Bromeliaceas: 306, 307. \\ Bromeliaceas: $306,307$. \\ Bromelias: 304, 305. \\ Bryologiam: 207, 208. \\ Bryologica: 141. \\ Buldophyllinae: 79. \\ Burmaniaceae: 298. \\ Burseracearum: 234. \\ Cactaceae: 64, 295. \\ Cactaceas: 178. \\ Cresalpinese: 27. \\ Calloideae: 117. \\ Cannaceae: 168. \\ Canellaceae: 222.
}


Canellaceas: 223.

Cipparidaceae: 106.

Caricoideae: 174.

Cutaretine: 293.

Cecrotinae: 79

Cropia: 309.

Celtis: 10.

Costrum: 124, 125.

Chlococceas: 213

Cinchoideae: 91.

Cladinoae: 193 .

Cocinae: 275 .

Corceas: 327.

Coloineae: 94.

Combrioideae: 119.

Commetaceas: 100

Commelinaceae: 301.

Concositae: 14, 15, 16, 17, 133.

Connamineae: 293.

Comaraceae: 13.

Cornophyta: 268.

Couscrare: 346.

Crestineae: 213.

Crotonea: 316.

Cucurbie: 211.

Cunonitaceae: 67, 83, 281.

Crathea: 53 : 108.

Crathea: 53.

Cymbiacea: 11.

Cyperacinae: 79.

Yperus: 175.

Ypridelinae: 73.

Granoideae: 17.

Cryptodium: 78, 79.

Oalbergiae: 26.

Dalechamp: 26.

Desmimpiae: 212.

Dichidaceen: 171.

Oichastalaceae: 266.

Diosconae: 82.

Oiostcoreacearum: 162.

Oiscoriaceae: 163.

Doryycetes: 256.

Orosopteris: 49.

Orymis; 221. 105, 279.

Echiloidea: 350

Erichoglassum: 48.

Erioceve: 190, 303.

Escallataceae: 166, 272.

Eupatoniae: 107.

Euphoriaceae: 15.

Euphorbiaceae: 211, 212, 230, 231, 232233234
Festucaceae: 91.

Fevilleae: 83.

Filices: $34,35,48$.

Fungi: 131.

Galeaceae: 27.

Gardenieae: 293.

Gelonieae: 231.

Gentianacese: 247.

Geonomese: 95.

Gesneriaceae: 139.

Gesneriaceen: 126, 127.

Gesniteae: 25.

Gomesas: 1.

Gongorinae: 79.

Gramineae: 89, 90, 91, 92.

Granatae: 30.

Griselina: 325.

Guettardeae: 213.

Guttiferae: 114.

Habenaria: 45.

Hamelieae: 293.

Hederaceae: 179.

Hedysareaceae: 17.

Hedytideae: 293.

Helianthoideae: 17.

Helenioideae: 17.

Hemelichen: 265.

Henriquezieae: 293.

Hepaticae: 154.

Hepaticarium: 317, 318, 319.

Hippomaneae: 212, 231.

Hordeaceae: 92.

Huntleyinae: 82.

Hymenophyllaceae: 323.

Hyophorbeae: 95.

Hyppericaceae: 257.

Icacineae: 110.

llicineae: 259.

Ingodiferae: 25.

Inuloidese: 16.

Iriarteae: 95.

Irideae: 160.

Ixoreae: 213.

Labiades: 38, 120.

Labiatae: 40, 42, 287.

Laellinae: $77,78$.

Lauraceae: 167, 191, 336.

Lecythidaceae: 30 .

Leguminosae: 25, 26, 27, 28.

Leguminosas: $147,148$.

Licheni: 273.

Lichenes: 214, 269.

Lichens: 276.

Lichenum: 267. 
Ligulatae: 17.

Lineae: 333.

Liparidinae: 74.

Liquens: 204.

Lobeliae: 159.

Loganiaceae: 248.

Loranthaceae: 101.

Loranthacearum: 270.

Lycopodiaceae: $217,218$.

Lycopodiaceas: 216.

Lycopodineae: 311.

Lythraceae: 161.

Magnoliaceae: 98.

Malpighiaceae: 135, 219, 220.

Malvacese: 137, 294.

Maranthaceae: 297.

Marcgraviaceae: 348.

Maxillaria: 12.

Maxillariinae: 94.

Melastomataceae: 68, 69, 70, 71, 72.

Meliaceae: 140, 344.

Melothrieae: 83.

Memecyleae: 72.

Menispermaceae: 88, 337.

Mercurialinae: 232.

Merianiae: 70.

Miconieae: 70, 71, 72.

Microlineae: 68.

Mimoseae: 27.

Monodrae: 169, 170.

Monimiacese: 240, $332,341$.

Monsteroideae: 117.

Moraceae: 60.

Mosses: 154.

Musci: 55, 155.

Muscorum: 205, 206.

Mussaendrae: 293.

Mutissiaceae: 17.

Myristicaceae: 340.

Myrsinaceae: 197, 202, 237.

Myrtaceae: 29, 30, 31.

Naucleceeae: 292

Neottinae: 73, 74.

Nyctaginaceae: 289.

Ochnaceas: 113.

Odontoglassse: 169.

Olacineae: 109.

Onagraceas: 199, 215.

Oncidlinae: 80, 82, 169.

Ophyrydinae: 73.

Orchidecese: $45,73,74,75,76,77,78,79,80,81,82,97,146,149,150,151,152,169,170,285$.

Orchideas: 286.

Orchidologiam: 226, 227, 228. 
Orchidologica: 143, 225, 228.

Oryzeae: 89.

Osmundaceae: 320.

Oxalidaceae: 165, 247.

Oxypetalum: $224,238$.

Paederieae: 292.

Palmae: $94,95$.

Palnicese: 90.

Papilionacearum: 25, 26.

Papilionadas: $147,148$.

Psppophoreae: 91 .

Passifloraceae: 186.

Phajinae: 78.

Phalarideae: 79.

Phaseoleae: 25 .

Philodendrinae: $118,119$.

Phyllantheae: 211.

Piperaceae: 86, 87.

Plantaginaceae: 244, 290.

Pleurothallidinae: $3,75,76$

Plukenthalis: 138.

Podosetunae: 233.

Polybotryaceas: 331.

Polygatrya: 50, 52.

Polypaceae: 188.

Polypociaceae: 12, 48, 49.

Polystium: 9.

Potamogetae: 74, 75.

Pothoigetonaceae: 7.

Proteaceae: 116, 117.

Pleudome: 157, 189.

Prolariaceae: 25 . 170.

Psycotriane: 25.

Preridieae: 213.

Pteridofita: 36.

Preridophyta: 42, 43, 65, 93.

eridophyteen: 282.

Rophieae: 94.

Rauwolfia: 193.

Rhamiphylleae: 213.

Rhexieas: 260.

Rhipsalis: 70.

Rodritis: 177.

Rosaceasia: 284.

Rubiace: 153

Rutaceas: 24, 213, 292, 293.

111.

Sabaleae: 95 .

Salvetia: 314.

Saniculoideae: 349.

apindaceae: $250,251,252,253,254,255$.
Sarcanthinae: 82.

Saxifragaceae: 235.

Schyzaeaceae: 322.

Schlumbegera: 178.

Scrophylarinae: 288.

Scrophulariaceae: 23.

Senecioneae: 59.

Senecionideae: 17.

Simplocaceae: 201.

Solana: 33.

Solanaceae: 315.

Sophoreae: 26.

Spermacoceae: 282.

Stellatae: 282.

Sterculiaceae: 278.

Stipaceae: 91.

Struthanti: 264.

Strychnos: 194.

Swartiziae: 27.

Syagrus: 58.

Symplocaceae: 54.

Tibouchineae: 69 .

Tilandsias: 4

Trifolieae: 25 .

Triuridaceae: 143, 296.

Typhaceae: 172.

Ulmaceae: 61.

Umbelliferae: 334, 349.

Urticaceae: 62.

Urticinae: 200.

Utricularias: 142.

Usneae: 204, 267.

Valerianaceae: 209.

Vicieae: 27.

Vellosieae: 229.

Verbenaceae: 271.

Verbobiaeae: 113.

Vernoniaceae: 14, 133.

Violaceae: 103.

Vochysia: 156, 314.

Vochysiaceae: $314,348$.

Winteraceae: 99, 338.

Xiridaceae: 5.

Xiridaceas: 308.

Zigocactus: 178.

Zygopetalinae: 79 .

Zingiberaceae: 241. 